\title{
Enhanced surveillance and investigation of coronavirus: what is required?
}

R.G. Pebody, ${ }^{1}$ A. Nicoll, ${ }^{2}$ U. Buchholz, ${ }^{3}$ M. Zambon ${ }^{1}$ and A. Mounts ${ }^{4}$

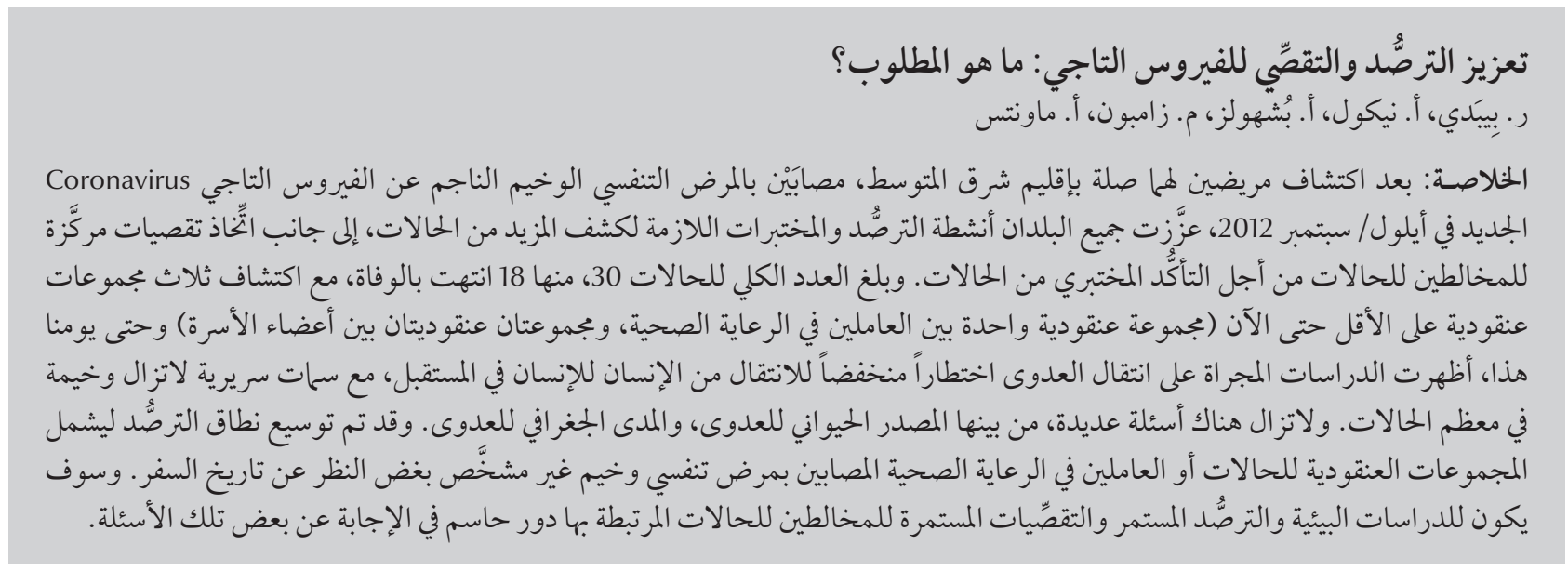

ABSTRACT Following the discovery in September 2012 of 2 patients, both with links to the Eastern Mediterranean Region, with serious respiratory illness due to novel coronavirus, all countries have instigated surveillance and laboratory activities to detect further cases, with intensive case-contact investigations undertaken on laboratory confirmation of cases. A total of 30 cases, of whom 18 have died, and at least 3 clusters have been detected to date (1 cluster among health-care workers and another 2 clusters among family members). To date, transmission studies have shown a low risk of onward human transmission, with clinical presentation remaining severe for the majority. Many questions remain including the zoonotic source and geographical extent of infection. Surveillance has been extended to include clusters of cases or health-care workers with severe, undiagnosed respiratory illness regardless of travel history. Environmental studies, on-going surveillance and linked casecontact investigations will provide a critical role in answering some of these issues.

\section{Surveillance et recherche renforcées pour le coronavirus : quels sont les besoins?}

RÉSUMÉ Suite à la découverte en septembre 2012 de deux patients atteints d'une maladie respiratoire sévère imputable à un nouveau coronavirus, tous deux ayant des liens avec la Région de la Méditerranée orientale, tous les pays ont initié des activités de laboratoire et de surveillance afin de dépister d'autres cas, et notamment des recherches de cas-contacts intensives pour les cas confirmés en laboratoire. Sur 30 cas au total, 18 sont décédés, et au moins trois groupes ont été identifiés à ce jour (un groupe chez des agents de soins de santé et deux autres groupes chez les membres des familles). À ce jour, les études de transmission ont démontré que le risque de future transmission interhumaine était faible, mais que le tableau clinique demeurait sévère pour la majorité des cas. II reste de nombreuses questions en suspens, notamment la source zoonotique et la propagation géographique de l'infection. La surveillance a été élargie à ces groupes de cas ou aux agents de soins de santé atteints de maladies respiratoires sévères non diagnostiquées indépendamment de leurs voyages passés. Des études environnementales, une surveillance continue et des recherches reliant des cas-contacts seront essentielles pour répondre à certaines de ces questions. 


\section{Introduction}

The discovery of a novel coronavirus $(\mathrm{nCoV})$ in a patient hospitalized with severe respiratory illness reported in September 2012 in Saudi Arabia [1] was shortly followed by the laboratory confirmation of nearly identical virus in a second individual in a hospital in London, United Kingdom [2]. This further, unlinked case had been transferred for medical care from Qatar following onset of a severe respiratory illness [3]. The detection of these 2 cases, both infected with a novel respiratory virus from the same genus as severe acute respiratory syndrome (SARS)-CoV, led to a global alert about the emergence of a new virus with possible SARS-like properties, with the experience of SARS at the forefront of response planning.

The appearance of a previously unrecognized virus, most closely related to beta coronaviruses in bats and which resulted in severe respiratory illness in humans, raised a series of important public health questions. These are summarized in the accompanying article by Nicoll et al. [4]. What was the likely animal reservoir of this virus? What were the risk factors for animal-to-human infection? What was the ability of the virus to spread from person-to-person? What was the extent of infection in the human population? And what was the spectrum of illness associated with infection in humans? The answers to all of these questions were unknown based on the experience of only 2 cases, 1 of which was fatal.

What role can surveillance play in helping to answer these questions? Surveillance was originally described by Langmuir as "the systematic collection, collation, analysis of data and dissemination of information on a specific disease to inform public health action" [5]. Latterly the epidemic intelligence role of surveillance, with timely alerting, analysis and dissemination of information, has gained greater prominence and is enshrined in the 2005 revision of the
World Health Organization (WHO) International Health Regulations [6,7].

The detection of the 2 initial cases, both of whom required intensive care support and who acquired their infection in the Eastern Mediterranean Region, together with the rapid development and availability of a sensitive and specific polymerase chain reaction (PCR) diagnostic tool for the detection of viral genetic material while virus shedding was still occurring [8], allowed the development of surveillance strategies to find further $\mathrm{nCoV}$ cases [9]. Relatively specific clinical case definitions were developed initially to provide a moderately good predictive value and ensure an efficient use of limited public health and laboratory capacity. The hierarchical case definitions developed by WHO and national organizations such as the Centers for Disease Control in the United States, Public Health England (formerly the Health Protection Agency) in England, the Robert Koch Institute in Germany and the European Centre for Disease Prevention and Control for the European Union [9-13] focused on the detection of individuals with severe undiagnosed respiratory illness with a history of travel from countries where indigenously acquired cases had been detected during the putative incubation period of nCoV. Such "patients under investigation" were recommended for $\mathrm{nCoV}$ testing (Table 1). Detailed laboratory algorithms needed to be established with a first-line sensitive assay to detect the virus, followed by a second-line assay to confirm virus detection, to avoid false conclusions about the incidence of disease

Early detection and isolation of laboratory-confirmed cases of symptomatic illness allows the rapid implementation of measures to limit potential spread of infection to close contacts. Case ascertainment also provides an opportunity for an in-depth epidemiological, clinical and virological investigation of the first cases and their close contacts using approaches developed during the 2009 influenza pandemic [11]. Collection of detailed information from cases (or their families when cases have died or were seriously unwell) allows a description of the demographic and clinical characteristics of cases and the development of hypotheses about risk factors for human infection from an as yet unknown reservoir. Describing laboratory-confirmed cases by time and place enables the epidemic curve and geographical distribution of this apparent emerging public health problem to be elaborated. Finally, the identification and follow-up of close contacts of confirmed cases provides the opportunity for detailed case-contact investigations that may provide clues to key epidemiological questions. These investigations involve intensive prospective follow-up of contacts for evidence of respiratory disease, together with virological and serological sampling. The availability of newly developed virological and serological assays provides the ability to detect secondary infections [14] and to measure the full disease spectrum among infected contacts [15].

These surveillance approaches, together with follow-up of an increasingly large number of close contacts, has resulted in 30 laboratory confirmed cases of $\mathrm{nCoV}$ being reported globally in the 6 months to 8 May 2013 [16]. Of the 30 confirmed cases, the majority have had severe respiratory disease, with 18 cases having died to date, a high case fatality rate. There is, however, a suggestion of a wider disease spectrum, with a small number of milder cases, detected through intense contact tracing activities [14], although active virological and serological follow-up of case-contacts regardless of symptoms has to date failed to uncover a significant hidden "iceberg" of mild or asymptomatic cases. The majority (24/30) of cases have been detected in the Eastern Mediterranean Region, with 6 cases diagnosed in Europe (the United Kingdom and Germany). Four of these latter 6 cases 


\begin{tabular}{|c|c|}
\hline $\begin{array}{l}\text { Organization } \\
\text { [reference] }\end{array}$ & Patient under investigation \\
\hline $\begin{array}{l}\text { World Health } \\
\text { Organization [9] }\end{array}$ & $\begin{array}{l}\text { A person with an acute respiratory infection, } \\
\text { which may include history of fever or } \\
\text { measured fever } \geq 38{ }^{\circ} \mathrm{C}\left(100.4^{\circ} \mathrm{F}\right) \text { and cough; } \\
\text { AND } \\
\text { Suspicion of pulmonary parenchymal disease } \\
\text { (e.g. pneumonia or acute respiratory distress } \\
\text { syndrome), based on clinical or radiological } \\
\text { evidence of consolidation; } \\
\text { AND } \\
\text { Residence in or history of travel to the Arabian } \\
\text { peninsula or neighbouring countries }{ }^{\mathrm{a}} \text { within } \\
10 \text { days before onset of illness; } \\
\text { AND } \\
\text { Not already explained by any other infection } \\
\text { or etiology, including all clinically indicated } \\
\text { tests for community-acquired pneumonia } \\
\text { according to local management guidelines. It } \\
\text { is not necessary to wait for all test results for } \\
\text { other pathogens before testing for nCoV. }\end{array}$ \\
\hline
\end{tabular}

Centers for Disease Control [10]
A person with an acute respiratory infection, which may include fever $\geq 38{ }^{\circ} \mathrm{C}\left(100.4^{\circ} \mathrm{F}\right)$ and cough;

AND

Suspicion of pulmonary parenchymal disease (e.g. pneumonia or acute respiratory distress syndrome based on clinical or radiological evidence of consolidation);

AND

History of travel from the Arabian peninsula or neighbouring countries ${ }^{\mathrm{a}}$ within 10 days; AND

Not already explained by any other infection or etiology, including all clinically indicated tests for community-acquired pneumonia ${ }^{b}$ according to local management guidelines.
Health Protection Any person with severe acute respiratory Agency [11]

infection:
Symptoms of fever $\geq 38^{\circ} \mathrm{C}$ or history of fever and cough;

AND

With evidence of pulmonary parenchymal disease (e.g. pneumonia or acute respiratory distress syndrome) based on clinical or radiological evidence;

AND

Not already explained by any other infection or etiology;

AND

History of travel to or residence in an area where infection with nCoV 2012 has recently been reported or where transmission could have occurred in the 10 days before onset of illness.
Other situations under investigation

\section{Ill contacts}

Individuals with acute respiratory illness of any degree of severity who, within 10 days before onset of illness, were in close physical contact with a confirmed or probable case of nCoV infection while the case was ill.

Any person who has had close contact with a probable or confirmed case while the probable or confirmed case was ill should be carefully monitored for the appearance of respiratory symptoms. If symptoms develop with the first 10 days after contact, the individual should be considered a "patient under investigation", regardless of the severity of illness, and investigated accordingly.

\section{Clusters}

Any cluster of severe acute respiratory infection, particularly clusters of patients requiring intensive care, without regard to place of residence or a history of travel; AND

Not already explained by any other infection or etiology, including all clinically indicated tests for communityacquired pneumonia according to local management guidelines.

Health-care workers

Health-care workers who care for patients with severe acute respiratory infections, particularly patients requiring intensive care, who develop unexplained pneumonia without regard to place or residence or history of travel.

Persons who develop severe acute lower respiratory illness of known etiology within 10 days after travel from the Arabian peninsula or neighbouring countries ${ }^{a}$ but do not respond to appropriate therapy;

OR

Persons who develop severe acute lower respiratory illness who are close contacts of a symptomatic traveller who developed fever and acute respiratory illness within 10 days after travel from the Arabian Peninsula neighbouring countries ${ }^{\text {a }}$. Close contact is defined as providing care for the ill traveller (e.g. a health-care worker or family member), or having similar close physical contact; or stayed at the same place (e.g. lived with, visited) as the traveller while the traveller was ill.

\section{Clusters of severe acute respiratory illness}

In addition, any clusters of severe acute respiratory illness in health-care workers in the United States should be thoroughly investigated. Occurrence of a severe acute respiratory illness cluster of unknown etiology should prompt immediate notification of local public health for further notification and testing.

Health-care worker based in intensive care unit (ICU) caring for patients with severe acute respiratory infection; OR

Close contact (i.e. prolonged face-to-face contact) during the 10 days before onset of illness with a confirmed case of $\mathrm{nCoV}$ infection while the case was ill.

Cluster

Two or more cases of severe acute respiratory infection requiring ICU admission, regardless of history of travel; AND

Not already explained by any other infection or etiology. 


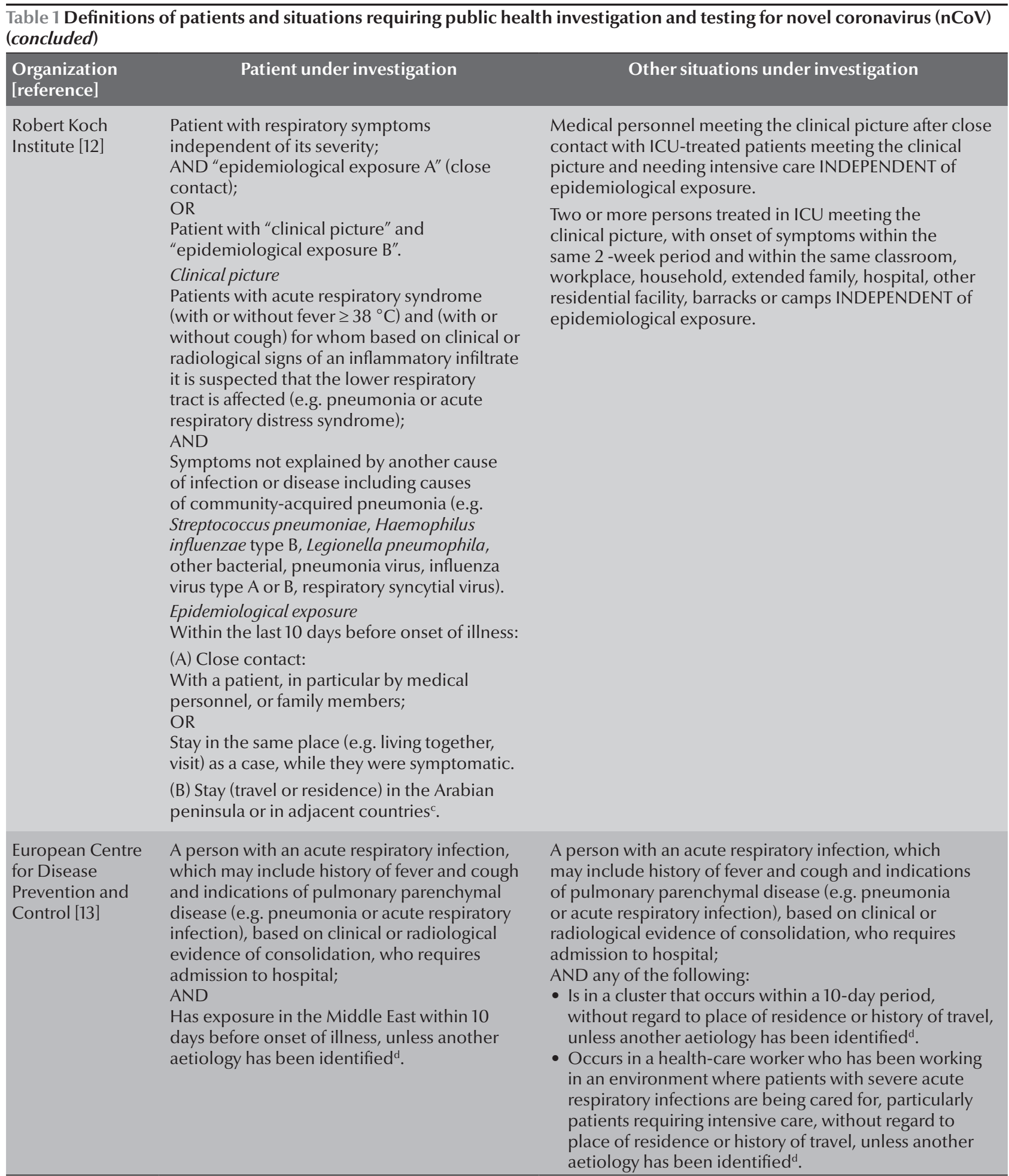

${ }^{a}$ Countries considered in the Arabian peninsula and neighbouring include: Bahrain, Iraq, Islamic Republic of Iran, Israel, Jordan, Kuwait, Lebanon, Oman, Palestinian territories, Qatar, Saudi Arabia, Syrian Arab Republic the United Arab Emirates and Yemen.

${ }^{b}$ Examples of respiratory pathogens causing community-acquired pneumonia include influenza A and B, respiratory syncytial virus, Streptococcus pneumoniae and Legionella pneumophila.

'Yemen, Qatar, Kuwait, Oman, Saudi Arabia, United Arab Emirates and Iraq, Jordan, Bahrain, Syrian Arab Republic, Lebanon, Islamic Republic of Iran, Palestinian Territories, Israel.

${ }^{d}$ Notes that dual infection has been demonstrated in at least 1 case and so that detection of another pathogen should not exclude testing for $n$ CoV where the first pathogen is unlikely to explain the clinical presentation). 
had travelled from the Eastern Mediterranean Region during the 10-day putative incubation period. The remaining 2 cases were both family members of one of the imported cases, who were infected following contact in the UK, one in a household setting, the other seemingly in a hospital setting [14]. Although the majority of cases have been sporadic, follow-up and investigation of close contacts of cases has identified likely evidence of non-sustained person-to-person transmission of virologically confirmed infection on at least 3 occasions. One cluster (of 2 confirmed cases and 11 probable cases) of severe respiratory illness among health care workers in a hospital in Jordan was identified based on retrospective testing; a second cluster (of 3 confirmed and 1 probable case) was identified in a family in Saudi Arabia and a third cluster (of 2 confirmed cases plus the index case) was found among family contacts of one of the confirmed cases imported to the UK. Investigation of each cluster did not demonstrate evidence of on-going person-to-person transmission. In May 2013, 13 confirmed cases were reported from Saudi Arabia to WHO and are currently under investigation [16].

The current picture is thus most likely of a severe, zoonotic infection with occasional transmission to humans, with limited person-to-person transmission. These preliminary and limited data suggest that the surveillance strategies implemented following the detection of the initial 2 cases are generally appropriate, with a focus on severe rather than mild disease to maintain optimum specificity and sensitivity, although many gaps in knowledge remain. Although onward infection in the human population has been limited to date, experience with SARS illustrated the possibility for super-spreading events to take place [17], highlighting the importance of ensuring timely detection of new cases through physician awareness and early testing and implementation of prevention and control measures. The ongoing detection of new cases linked (directly or indirectly) to the Eastern Mediterranean Region, highlights the importance of continuing surveillance for cases of severe respiratory disease with a history of travel from countries where sporadic cases have been reported (Table 1). Global and national $\mathrm{nCoV}$ surveillance and investigation activities have evolved based on the emerging epidemiological picture. WHO has adapted $\mathrm{nCoV}$ surveillance guidelines with a focus on detecting the full geographical extent of cases and to detect evidence of person-to-person transmission. Key changes to the case definition will allow the full geographical "reach" of the infection in the human population to be better delineated by extending previous surveillance case definitions to include clusters of severe undiagnosed respiratory disease and severe undiagnosed respiratory disease in health-care workers regardless of history of travel (Table 1). Continuing to undertake case-contact transmission studies of new cases and follow-up of contacts of newly confirmed cases is essential to detect evidence of personto-person transmission. Investigation protocols and guidelines for these case-contact investigations have been developed [11] and should be deployed for future confirmed cases $[11,18]$. The emergence of $\mathrm{nCoV}$ has also highlighted the importance of international collaboration between laboratories and countries where suspect cases have occurred. This has allowed the technology transfer of real-time PCR diagnostic tools. The serological diagnostics for $\mathrm{nCoV}$ remain technically challenging, and the importance of a global reference laboratory will remain vital.

Such surveillance activities need to be supported by applied epidemiological studies to answer more specific questions and which should be undertaken in a standardized fashion to allow global pooling of results [13]. Other studies include: environmental and animal studies to identify the source of the infection; serological studies in a range of potentially exposed populations; case-control studies to identify and quantify risk factors for animal-toperson transmission and retrospective testing of sample repositories from cases of severe undiagnosed respiratory illness in a range of geographical settings. These studies should provide answers to the source of $\mathrm{nCoV}$, whether $\mathrm{nCoV}$ is occurring in other settings, how long it has been around and what burden of illness it is responsible for. Such information is key to informing the revision and optimization of public health prevention and control measures to reduce the burden of disease due to this novel infection.

\section{References}

1. Zaki AM et al. Isolation of a novel coronavirus from a man with pneumonia in Saudi Arabia. New England Journal of Medicine, 2012, 367:1814-1820.

2. Bermingham A et al. Severe respiratory illness caused by a novel coronavirus, in a patient transferred to the United Kingdom from the Middle East, September 2012. Eurosurveillance, 2012, 17(40):pii 20290.

3. Pebody RG et al. The United Kingdom public health response to an imported laboratory confirmed case of a novel coronavirus in September 2012. Eurosurveillance, 2012, 17(40):pii 202922.
4. Nicoll A. Public health investigations required for protecting the population against novel coronaviruses. Eastern Mediterranean Health Journal, 2013, 19(Suppl.):S61-S67.

5. Langmuir AD. The surveillance of communicable diseases of national importance. New England Journal of Medicine, 1963, 268:182-192.

6. International health regulations (2005), 2nd ed. Geneva, World Health Organization, 2005.

7. Heymann D, Mackenzie J, Peiris M. SARS legacy: outbreak reporting is expected and respected. Lancet, 2013, 381:779-781. 
8. Corman VM et al. Assays for laboratory confirmation of novel human coronavirus (hCoV-EMC) infections. Eurosurveillance, 2012, 17(39):pii 20285.

9. Interim surveillance recommendations for human infection with novel coronavirus (28 November 2012). Geneva, World Health Organization, 2012 (http://www.who.int/csr/disease/coronavirus_infections/InterimRevisedSurveillanceRecommendations_nCoVinfection_20121128.pdf, accessed 6 May 2013).

10. Coronavirus. Update, case definitions, and guidance. Centers for Disease Control and Prevention [online factsheet] (http:// www.cdc.gov/coronavirus/ncv/case-def.html\#case, accessed 6 May 2013).

11. The first few hundred (FF100). Enhanced case and contact protocol v4.0. Epidemiological protocols for comprehensive assessment of early novel coronavirus cases and their close contacts in the United Kingdom. London, Health Protection Agency, 2012 (http://www.hpa.org.uk/webc/HPAwebFile/ HPAweb_C/131713630080, accessed 6 May 2013).

12. Schwere respiratorische Erkrankungen in Verbindung mit einem Neuartigen Coronavirus. Falldefinition zur Fallfindung, Meldung und Übermittlung. Aktualisierte Version vom 12.12.201 [Severe respiratory disease in conjunction with a novel coronavirus. Case definition for case finding, reporting and communication. Updated version 12.12.201]. Berlin, Robert Koch Institute, 2012 (http:// www.rki.de/DE/Content/InfAZ/C/Corona/Corona_Falldefinition.pdf?_blob=publicationFile, accessed 6 May 2013).
13. Rapid risk assessment. Severe respiratory disease associated with a novel coronavirus. 19 February 2013. Stockholm, European Centre for Disease Control and Prevention, 2013 (http://www. ecdc.europa.eu/en/publications/Publications/novel-coronavirus-rapid-risk-assessment-update.pdf, accessed 6 May 2013).

14. Health Protection Agency (HPA) UK NCoV Investigation team. Evidence of person-to-person transmission within a family cluster of novel coronavirus infections, United Kingdom, February 2013. Eurosurveillance, 2013, 18(11):pii 20427.

15. Buchholz $U$ et al. Contact investigation of a case of human novel coronavirus infection treated in a German hospital, October-November 2012. Eurosurveillance, 2013, 18(8):pii 20406.

16. Novel coronavirus infection-update. 8 May 2013. World Health Organization [online] (http://www.who.int/csr/disease/ coronavirus_infections/update_20130508/en/index.html, accessed 8 May 2013).

17. Peiris JS et al. The severe acute respiratory syndrome. New England Journal of Medicine, 2003, 349:2431-2441.

18. Van Kerkhove $\mathrm{M}$ et al. The consortium for the standardization of influenza seroepidemiology (CONSISE): a global partnership to standardize influenza seroepidemiology and develop influenza investigation protocols to inform public health policy. Influenza and Other Respiratory Viruses, 2013, 7(3):231-234. 